\title{
Smartphone Household Wireless Electroencephalogram Hat
}

\author{
Harold Szu, ${ }^{1}$ Charles Hsu, ${ }^{2}$ Gyu Moon, ${ }^{3}$ Takeshi Yamakawa, ${ }^{4}$ \\ Binh Q. Tran, ${ }^{1}$ Tzyy Ping Jung, ${ }^{5}$ and Joseph Landa ${ }^{6}$ \\ ${ }^{1}$ Department of Biomedical Engineering, The Catholic University of America, Washington, DC 20064, USA \\ 2 Trident Systems Inc., Fairfax, VA 22030, USA \\ ${ }^{3}$ Department of Electronic Engineering, Hallym University, Chuncheon, Gangwon-do 200-702, Republic of Korea \\ ${ }^{4}$ Fuzzy Logic System Institute, Semiconductor Center, Kitakyushu Science and Research Park, Kitakyushu 808-0135, Fukuoka, Japan \\ ${ }^{5}$ Swartz Center, University of California, San Diego, CA 92093, USA \\ ${ }^{6}$ Briartek Inc., Alexandria, VA 22301, USA
}

Correspondence should be addressed to Harold Szu; szuharoldh@gmail.com

Received 25 May 2012; Accepted 21 October 2012

Academic Editor: Soo-Young Lee

Copyright (C) 2013 Harold Szu et al. This is an open access article distributed under the Creative Commons Attribution License, which permits unrestricted use, distribution, and reproduction in any medium, provided the original work is properly cited.

Rudimentary brain machine interface has existed for the gaming industry. Here, we propose a wireless, real-time, and smartphonebased electroencephalogram (EEG) system for homecare applications. The system uses high-density dry electrodes and compressive sensing strategies to overcome conflicting requirements between spatial electrode density, temporal resolution, and spatiotemporal throughput rate. Spatial sparseness is addressed by close proximity between active electrodes and desired source locations and using an adaptive selection of $N$ active among $10 N$ passive electrodes to form $m$-organized random linear combinations of readouts, $m \ll N \ll 10 N$. Temporal sparseness is addressed via parallel frame differences in hardware. During the design phase, we took tethered laboratory EEG dataset and applied fuzzy logic to compute (a) spatiotemporal average of larger magnitude EEG data centers in 0.3 second intervals and (b) inside brainwave sources by Independent Component Analysis blind deconvolution without knowing the impulse response function. Our main contributions are the fidelity of quality wireless EEG data compared to original tethered data and the speed of compressive image recovery. We have compared our recovery of ill-posed inverse data against results using Block Sparse Code. Future work includes development of strategies to filter unwanted artifact from high-density EEGs (i.e., facial muscle-related events and wireless environmental electromagnetic interferences).

\section{Introduction}

A noninvasive electrical response exists near the scalp from neuron ionic transmission among neural network and may be measured via electroencephalography (EEG). Wang and colleagues of UCSD $[1,2]$ have demonstrated the efficacy of an untethered, wireless brain machine interface (BMI) system using 20 dry electrodes embedded into a head cap. The wireless EEG head cap system has a built-in bandwidth filter for eliminating environmental noise, for example, $60 \mathrm{~Hz}$ household utility line and also for pattern noise. Additionally, this pattern noise filter naturally represents a neuron threshold logic which can be used for assessment of cognitive function and for diagnosis.
Figure 1 shows various observed EEG state, representative EEG patterns, typical frequency ranges, correlation to different activities, and state of mind/levels of engagement. There is a simple rule of thumb about the mnemonics of brainwaves in terms of " $D, T, A, B$ " phonetic equivalence with "deep tap." "Deep tap" separates apart $D=\operatorname{delta}(0-4 \mathrm{~Hz}) ; T=$ theta $(4-7 \mathrm{~Hz}) ; A=$ alpha $(8-12 \mathrm{~Hz}) ; B=$ beta $(13-30 \mathrm{~Hz})$ at about $4 \mathrm{~Hz}$ or more intervals. In classical neuropsychological studies, the central nervous system (CNS) is involved in the information processing of massively parallel and distributive associative memory stored at the hippocampus. This gives rise to an event-related potential (ERP) at latency greater than 300 milliseconds, or 0.3 second delay, often referred to as P300 brainwave component. 


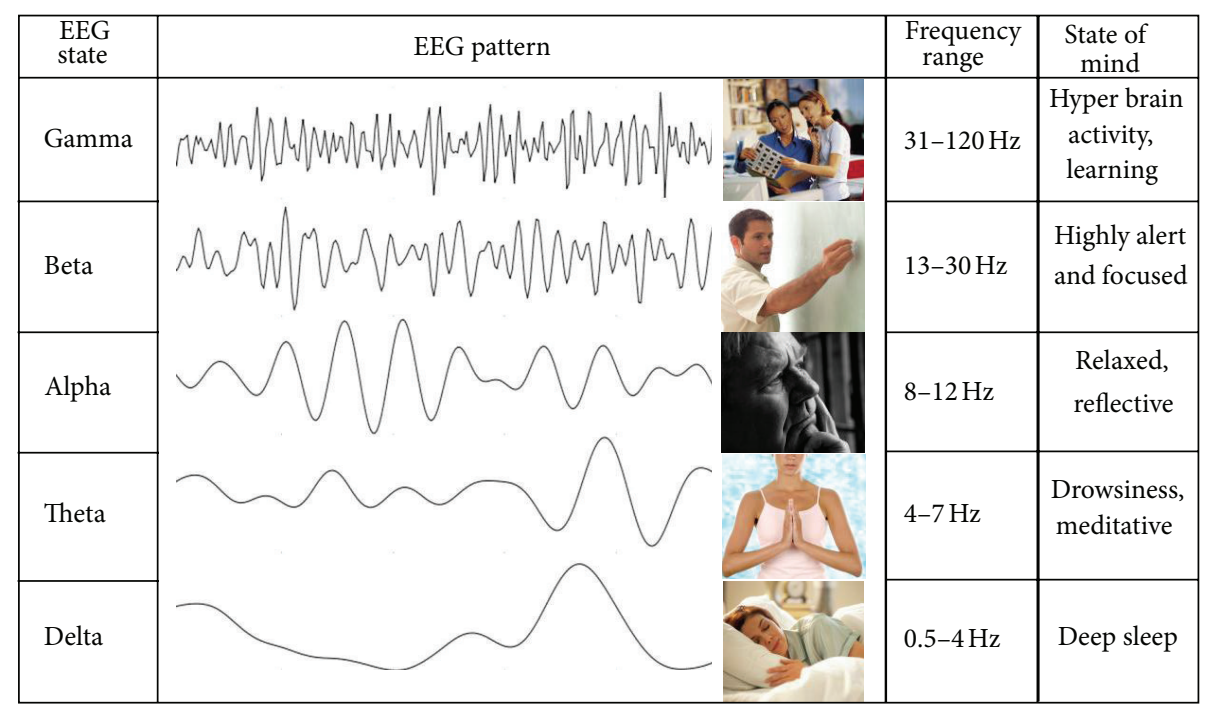

FIGURE 1: Typical EEG brainwavepatterns during various activities and cognitive states.

Compressive sensing (CS) techniques for dealing with sparse problem sets have been developed by Candès et al. [3, 4] and Donoho [5] (CRT\&D) in 2007. Making a sparse $m$-linear combination, $m \approx 1.3 k \ll N$, CRT\&D proved a restricted isometry property theorem that permits an equivalent $L_{1}$-sparse source condition for a noisy LMS $L_{2}$-similarity. They adopt a purely random compressive sensing matrix $[\Phi]_{m, N}$ of the size of $m$-rows and $N$-columns and then solve by postprocessing a linear programming algorithm to invert an underdetermined linear $m$-combination of " $N$ " data in a sparse Wavelet Transform representation $[\Psi]_{N, k}$. We wish to modify their mathematical general case to a special design of $[\Phi]_{m, N}$. We apply another well-known Independent Component Analysis (ICA) strategy whereby solving both the unknown mixing matrix $[\mathrm{ICA}]_{m, k}$ and the unknown source $\vec{s}$ by means of blind source separation (BSS). Using this technique, we can specially design an organized sparse sensing matrix $\left[\Phi_{s}\right]_{m, N}$ to increase SNR to take advantage of the proximity effect by physically placing sensing elements at source locations [5]. We can then further enhance the degree of sparseness by video temporal frame differencing at the electrode sensor domain.

Compressive sensing (CS) is different from JPEG or JPEG2000 postprocessing compression. In a similar fashion to how a focal plane array CCD outputs from pixels sensors with $m$ linear combinations of all $N$ sensor data $(m \ll N$ underdetermined), the advantage of a true medical CS value is that it can be obtained more quickly and at a less exposure (i.e., radiation) to patients. This ill-posed inversion is mathematically possible, because CRT\&D proved a CS theorem that the linear combinations readouts coefficients form a purely random bipolar analog CS sampling matrix [Ф] that is pseudoorthogonal among $m$ rows, where $m \cong$ $1.3 k$ corresponding to the intrinsic sparse degree-of-freedom of the information. In other words, small analog coefficients will be zeroed out, or the locations of $N$ data will not send any $X$-ray radiation through the patients in the first place. Besides optical imaging, no one has previously demonstrated CS in the $X$-ray domain.

The overall goal of this work, shown in Figure 2, is to develop an easy-to-use wireless, portable smartphone-based EEG system for at-home measures of cognitive function with the same accuracy and resolution of in-clinic measures of brain function.

\section{Strategy of Generating Sparseness}

To be successful in applying CS techniques, the sparse data acquisition strategy deserves a special attention. Usually, data acquisition intrinsically restricts how the sensor-material interaction generates spatiotemporal sampling. This elementary chemical-physics interaction is not flexible and cannot be easily manipulated into another representation where the intrinsic degree of freedom manifests clearly into a numerical quantization value. Without detailed knowledge of sensordata interaction, the tricks of revealing itself as sparse information content are limited as follows. We consider first the mathematical transform to concentrate the spatiotemporal information and therefore reduce the apparent sparseness. Then, one considers how to embed the mathematics into sensor circuitry hardware, if possible.

(1) Event Correlation Domain. If the data is dense, the correlation of data might not be. After a proper threshold, the correlation, for example, motion target indicator (MTI), is sparse.

(2) Frequency Transform Domain. The histogram could be quantized differently into sparse resolution. Fourier transform frequency domain of a band-limited function requires the Gerchberg-Saxon-Papoulis super-resolution iteration algorithm in order to recover a postcompression algorithm. This is needed to shift to the sensor domain so as to manifest its data sparseness for CS implementation. 

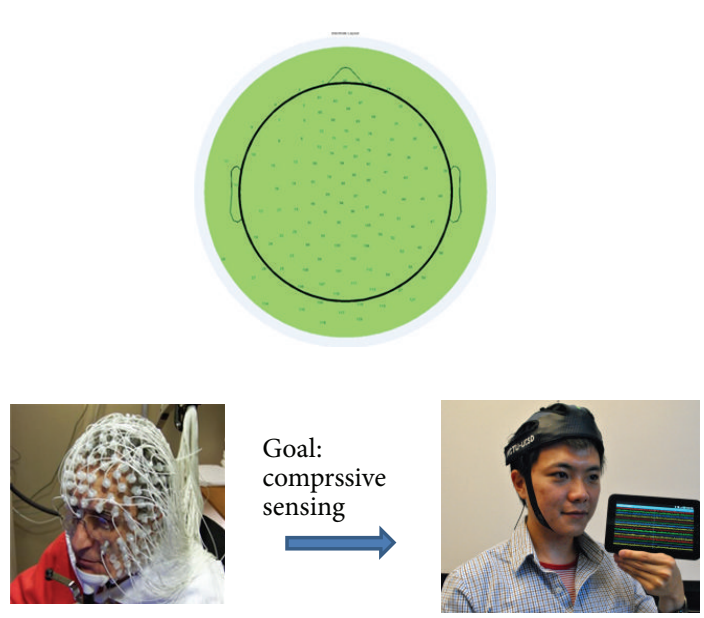

FIGURE 2: Proposed system for wireless wearable, cap-based high spatial density EEG intended for at-home use (right) to replace clinic-based, tethered EEG system (left).

(3) Time-Frequency Joint Representation (TFJR). Optimum concentration of information can be demonstrated using TFJR, Short-Time Fourier Transform, Wigner Distribution Function (frequency charge weighted convolution), and Woodward Ambiguity Function (Doppler shifted correlation).

(4) Frame Differencing Change Detection (FDCD). Often an oversampled video allows for redundancy reduction by a simple frame differencing.

(5) Sensor-Sensing Proximity Effect (SSPE) and Blind Source Separation (BSS). See the following theorem (Section 3).

We generate both spatial and temporal degrees of sparseness in EEG separately in (i) and (ii) as follows: (i) spatial sparseness: by enhancing intrinsic SNR by the closeness proximity between active electrodes and the desired EEG source locations, using an adaptive software-controlled selection of $N$ active, among $10 N$ passive, electrodes to form $m$-organized random linear combinations of readouts, $m \ll N \ll 10 N$; (ii) temporal sparseness: massively parallel frame difference by means of on-board-computing on each electrode by sampling and hold low-power mixed-signal difference circuitry.

For example, during the design phase, we took the tethered laboratory EEG data set (see Figure 3), applying fuzzy logic to compute (a) spatiotemporal average of larger magnitude of EEG data centers in 0.3 second (i.e., Event Related Potential P300 brainwave) and (b) inside brainwave sources by Independent Component Analysis (ICA) blind deconvolution, without the need of knowing the impulse response function mixing matrix. Consequently, we can choose those active linear-combination read-out electrodes by software selection, as if we had tailored a nonuniform set of electrode distribution at exactly fuzzy-centered locations of sources for enhancing SNR and therefore the degree of sparseness by a simple band-passing threshold.

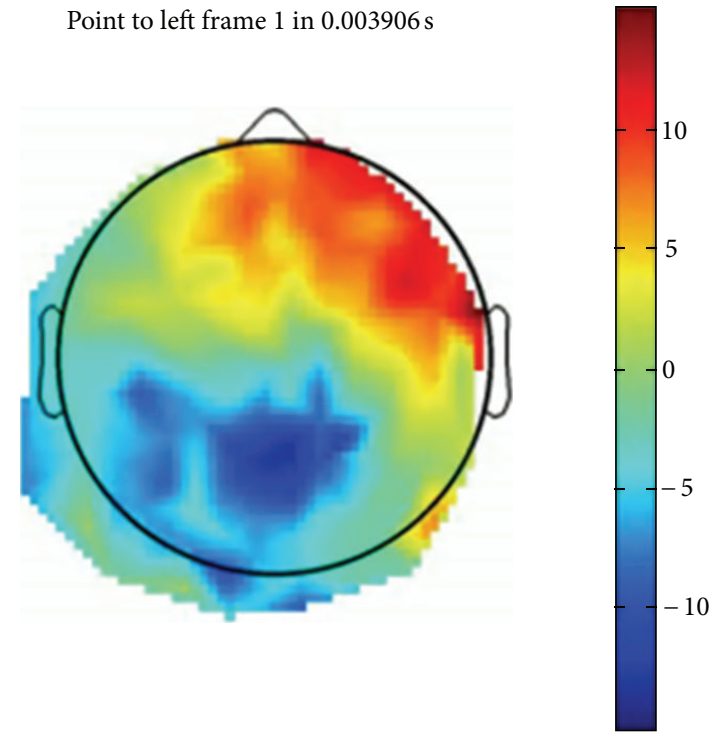

FIGURE 3: Mapping of spatiotemporal brainwave dynamics in highdensity tethered lab measurement.

\section{Theorem: Enhance the Sparseness by ICA}

Given a CRT\&D-defined purely random CS $[\Phi]_{m, N}$, we can permute column vectors maintaining the pseudoorthogonality and yet produce an organized CS $\left[\Phi_{s}\right]_{m, N}$ according to sensor-sensing proximity effect as follows:

$$
\begin{aligned}
& \text { Input } R^{N}: \vec{X}=\sum_{n=1}^{N} s_{n} \Psi_{n}^{T} \cong \sum_{n_{k}=1}^{k} s_{n_{k}} \Psi_{n_{k}}^{T}=[\Psi]_{N, k} \vec{s}, \\
& \text { Output } R^{m}: \vec{Y}=\sum_{m=1}^{M} x_{m} \Phi_{m}^{T}=[\Phi]_{m, N} \vec{X}, \\
& \text { ICA } R^{m}: \vec{Y}=[\Phi]_{m, N}[\Psi]_{N, k} \vec{s}=[\mathrm{ICA}]_{m, k} \vec{s}, \\
& {\left[\Phi_{s}\right]_{m, N} \cong[\mathrm{ICA}]_{m, k}[\Psi]_{N, k}^{-1} .}
\end{aligned}
$$


In Figures 4 and 5, we illustrated the difference between CRT\&D CS versus our video CS. The difference may be rooted in the method of producing information concentration. Unlike previous applications that concentrated on generating a single-frame sparseness, we applied the video change detection to produce a naturally sparseness. While CRT\&D generated the information concentration by some favored transform (FFT or DWT) domain, we worked on a spatiotemporal information processing using the change detection by difference frames. Two resulting sampling matrices may be shown to be equivalent in the sense that a full row or column permutations will not alter the original matrix determinant, and thus we can permute the nonzero value pixels of CRT\&D template in Figure 4 to the significant change pixels' location, for example, smiling face. This is to illustrate the potential rank equivalence between CRT\&D purely random matrix and our organized pseudorandom matrix. In our video CS approach, we constructed directly the sampling pixels through frame differences, without the aforementioned detouring. The benefit of our organized sparseness may be measured by a good quality of video sending through a narrow bandwidth of smart phone in Figure 6.

\section{Hardware and Software Approach}

Our improvement is mainly due to aforementioned enhancing SNR for the manifest of intrinsic sparseness using the physical sensor-sensing proximity mechanism. Secondly, we apply the temporal oversampling video nature to reduce the sparseness between frames furthermore by reducing the redundancy using the neighborhood frame differencing. Hardware implementation is illustrated by Simulation Program with Integrated Circuit Emphasis SPICE as shown in Figure 6.

In Figure 6, semiconductors have 3 ports: anode, cathode, and a gate. The gate has two ports, left and right lower corners, denoted by $V_{1}$ and $V_{2}$. Gate potentials are taken sequentially from an electric potential detector for the prior and subsequent potential intensities. The difference in the output current, $I_{\text {out }}$, located at the right-hand side middle portion is obtained by locally and adaptively modifying the time span between detectors $V_{1}$ and $V_{2}$ according to current EEG potential and its early EEG potential.

\section{Modeling and Simulation}

We can iteratively recover the compressive sensing image data, because we have enhanced SNR intrinsically by a proper placement of electrodes close to desired brainwave sources reducing the apparent degree of sparseness by threshold. The apparent sparseness is further reduced by a smart video data change acquisition strategy in the sensor electrode domain. For spatial sparseness, we concentrated the measurement electrodes at known desired positions of brainwave sources, reducing further the contact impedance of each electrode using a gecko-like directional nano-grip conducting surface. Toward temporal sparseness, we note that the originally CRT\&D CS algorithm has $\mathrm{m}$ pseudoorthogonal

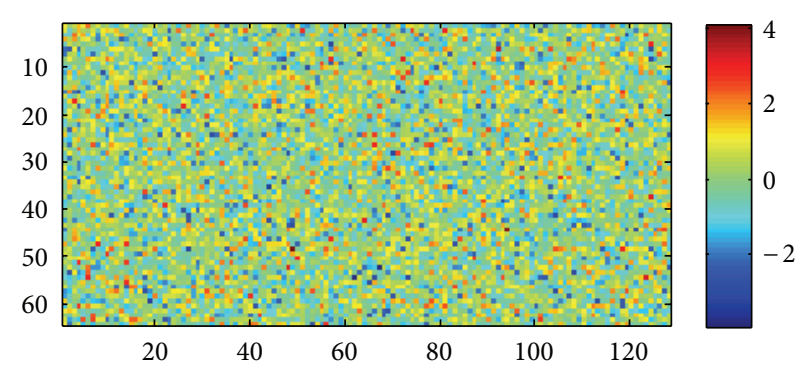

FIGURE 4: CRT\&D 2:1 ratio compressive sensing coefficients generated by uniformly random bipolar analog values for linear combination of measured data value $N=128$ and $m=64$.

linear combinations forming the sparse sensing matrix $[\Phi]$ of the size $m \times N$, where $m \ll N$. We moved those larger $L_{1}$ norm column vectors of $[\Phi]$ as wholes to desired brainwave sources, overlaid with nonuniform designed locations of electrodes. In short, by rearranging the column vector as a whole, we did not disturb the inner products among any two rows of $[\Phi]$. The degree of sparseness can be increased serendipitously if electrodes are properly placed at desired brainwave sources. Then, the physics and the geometry of our nonuniform sampling can concentrate the desired brainwave measurement, naturally generating the source and the weighted-measurement proximity effect, so that the larger SNR is coincided with the larger weight coefficient of $\left[\Phi_{S}\right]$. This procedure completed our theory of organized sensing matri $\left[\Phi_{S}\right][6]$.

Figures 7 and 8 show representative data using the proposed CS algorithm (column 2) for measurement of spatiotemporal brainwave dynamics as compared to clinic-based EEG measures (column 1). The differences between the original and CS images are shown in the 3rd column of Figures 7 and 8.

\section{Conclusion}

An at-home EEG system capable of real-time monitoring has several potential health monitoring applications, particularly for aging populations who desire to age-in-place as well as those living with acute health conditions. Utilization of EEG for detecting convulsive [7-9] and nonconvulsive [10, 11] epilepsy is well developed and documented in the literature with sensitivities between $80 \%-90 \%$. Nonconvulsive seizures may mimic EEG patterns seen in encephalopathy's, that is, diminished states of consciousness. Real-time EEG monitoring during sleep may provide valuable information on alterations in sleep patterns, which has been demonstrated to be effective in detecting and predicting anxiety, depression, and early dementia in the elderly [12-15]. Anxiety and depression have been directly correlated to numerous deleterious chronic health conditions such as cardiovascular disease, stroke, sleep disorders, and diabetes to name a few. In many cases, medications prescribed in response to these conditions may cause the onset of depression in patients. Having a mechanism to detect and predict onset of anxiety and depression would be a powerful out-of-clinic tool. 


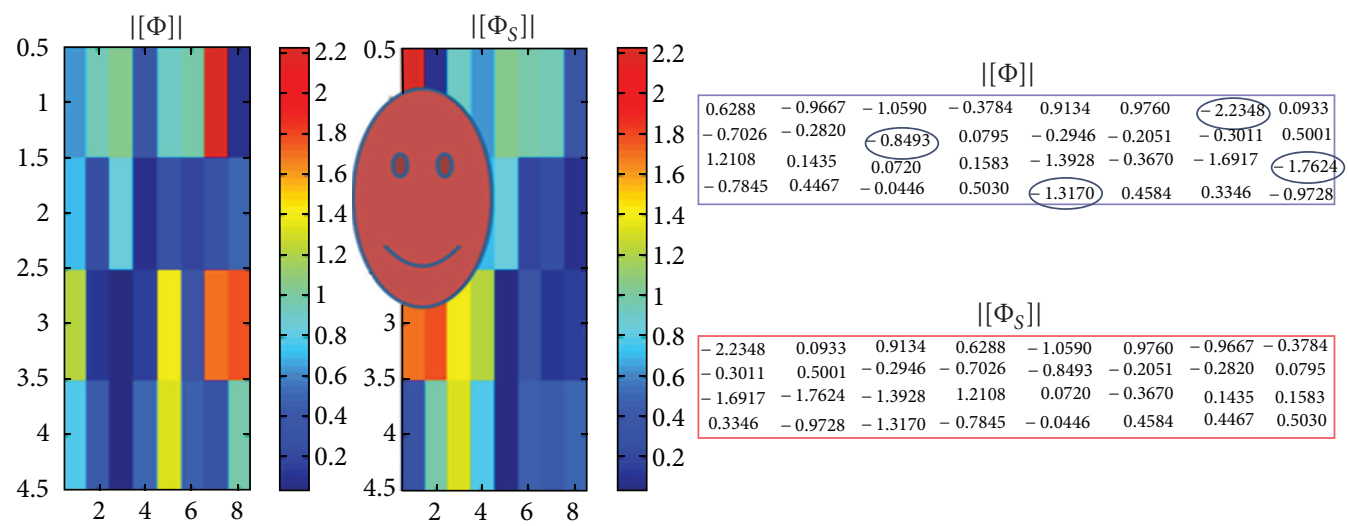

FIGURE 5: Illustration of whole column-wise permutations to move the larger column vector of $l_{1}$ norm to a specific location, for example, the left top corner, where the brainwave sources are known to be located.

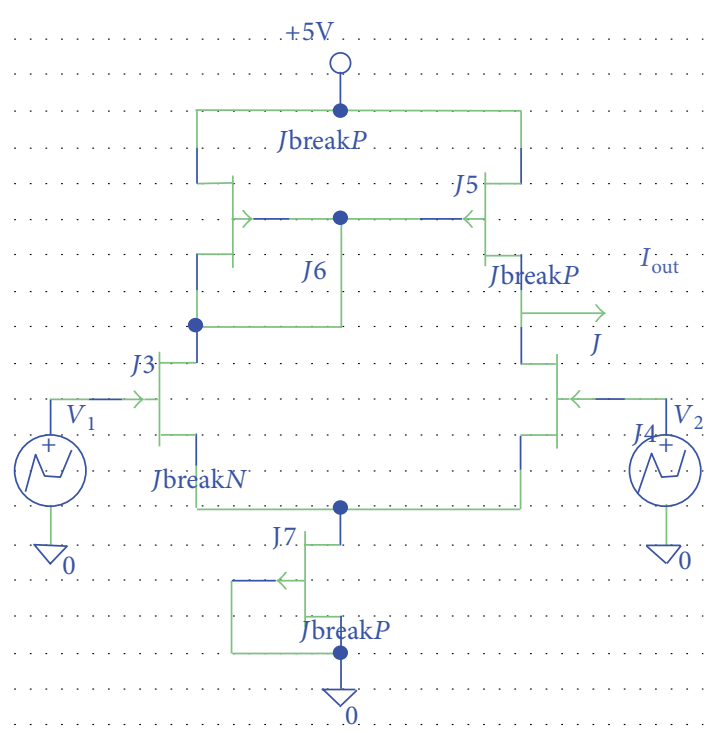

(a)

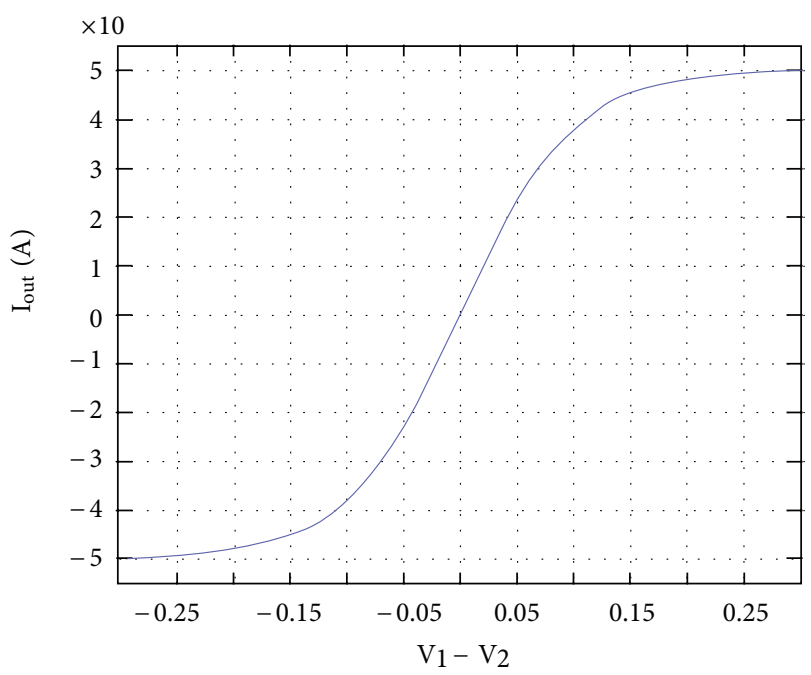

(b)

FIGURE 6: SPICE implementation of the working CS strategy.
However, further research efforts are required to enhance specificity and sensitivity of EEG for these applications as drowsiness and response to medications have been shown to directly affect EEG patterns. A potential application for athome EEG monitoring includes assessment of head injuries and postconcussion syndrome. As these conditions affect the alertness of individuals, there is evidence that head injuries affect alpha wave patterns of the EEG in the range of $8-10 \mathrm{~Hz}$ [16-18].

While many of the previously discussed studies have demonstrated the potential for EEG to detect and assess cognitive impairments, most have been conducted under controlled research conditions in the clinic. Extensions of these findings to the home require further investigation as environmental conditions are likely to affect EEG data collected. The impact of alertness/drowsiness is a confounding factor that requires further study as well as psychotropic medications that may be prescribed to consumers. Further developments in automated EEG analyses and data reduction techniques, as well as event recording for future analysis, are required to manage the vast amounts of data collected from the proposed system.

Scalp EEG (i.e., EEG from electrodes over the skin and hair through electrically conductive paste) is often affected by a variety of unwanted artifact such as (i) EMG, EOG, and so forth, (ii) biologically-related signal distortion by the skull bone and skin, and (iii) environmental noise and electromagnetic interference (i.e., $60 \mathrm{~Hz}$ powerline, computers, and some other electric equipment). Thus, the scalp EEG is often unstable and unreliable, even in a shielded space, which leads to misidentification of the position and volume of the epileptogenic focus and other cognitive measures. Ideally, we wish to improve our strategies and proposed system to more accurately measure electrocorticography (ECoG). Future improvements will focus on a CS module of high-density dry electrodes with nano-grid surface, thereby reducing contact impedance of the original inconvenient EEG measuring wet electrodes. Two spatiotemporal analog filters at electrode level will be implemented to eliminate both the unstable motion-generated EMG and wireless environmental electromagnetic interference (we-EMI). Such 
Original plot, $0.015625 \mathrm{~s}$
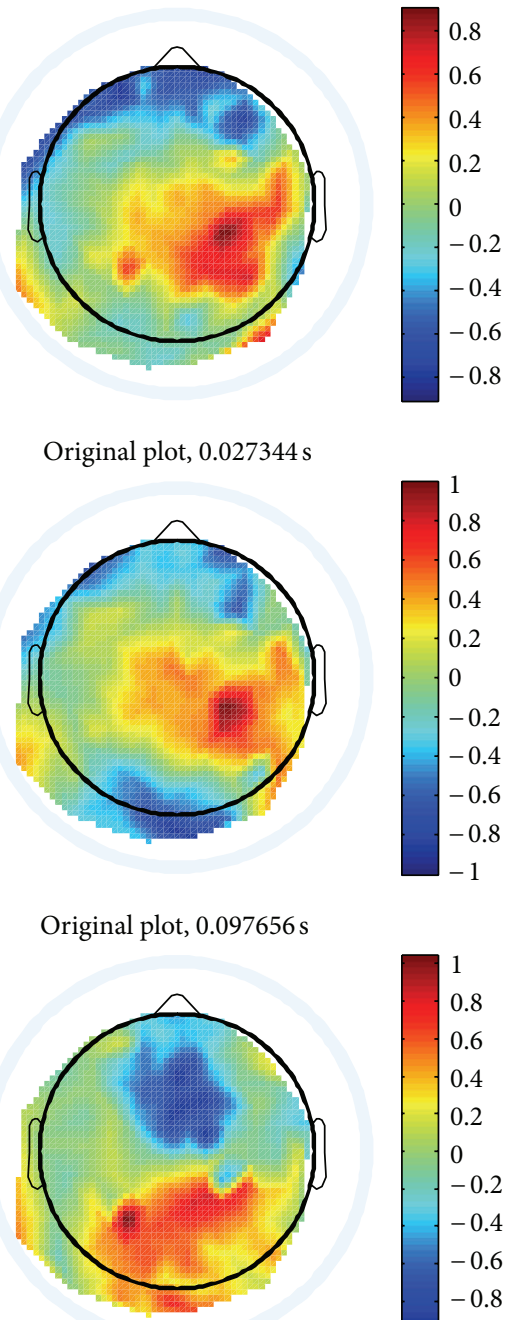

Original plot, $0.12109 \mathrm{~s}$

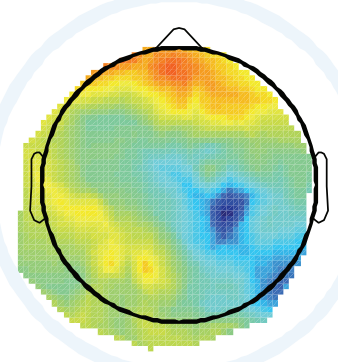

CS plot, $0.015625 \mathrm{~s}$

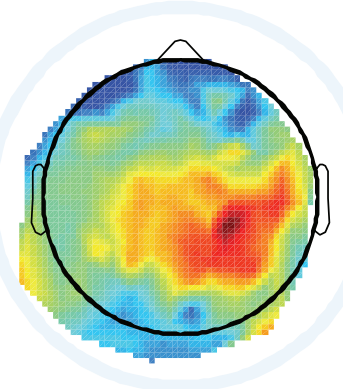

CS plot, $0.027344 \mathrm{~s}$

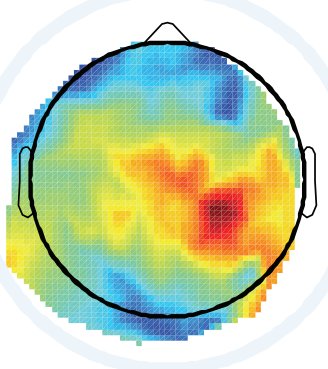

CS plot, $0.097656 \mathrm{~s}$

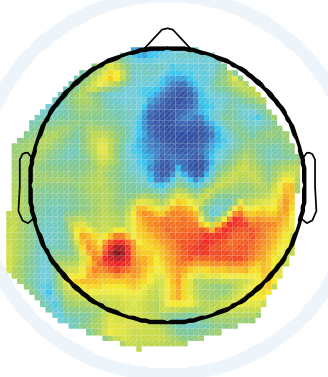

CS plot, $0.12109 \mathrm{~s}$

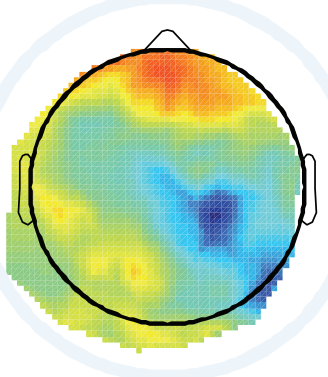

Difference $\mathrm{b} / \mathrm{w}$ original and CS, $0.015625 \mathrm{~s}$
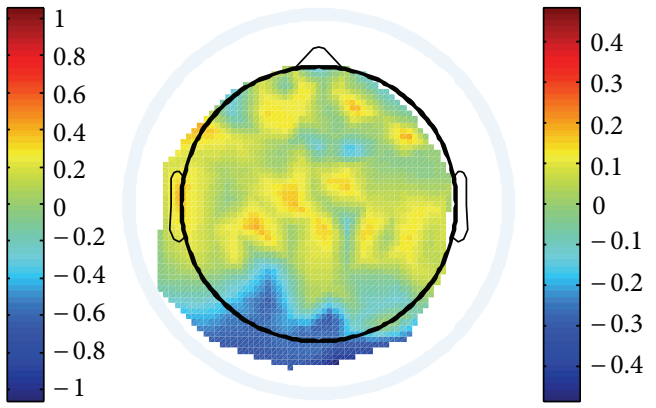

Difference b/w original and CS, $0.027344 \mathrm{~s}$
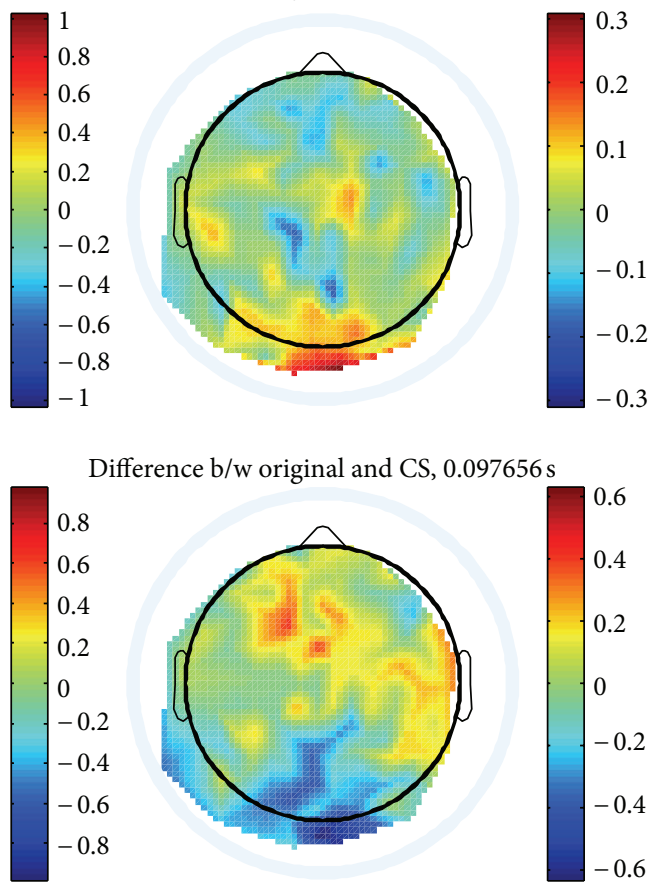

Difference b/w original and CS, $0.097656 \mathrm{~s}$

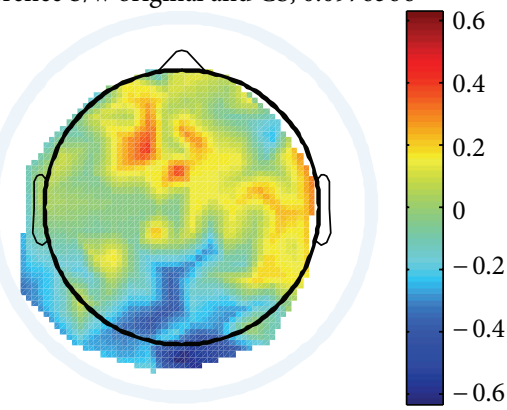

Difference b/w original and CS, $0.12109 \mathrm{~s}$
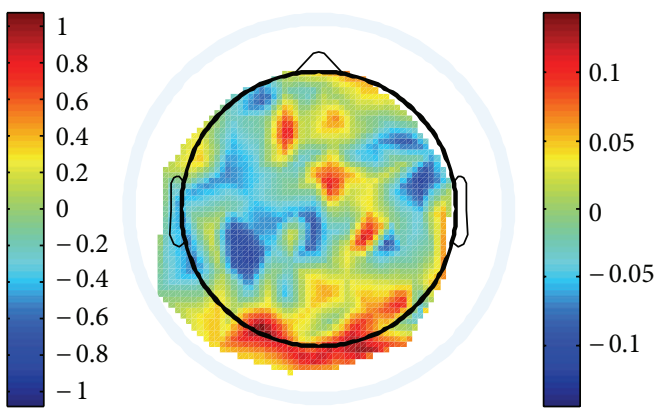

Figure 7: Comparison of clinic-based EEG data versus CS algorithm. Number of frames: 256 ( 1 sec.), $M=64, N=128$, compression ratio = $2: 1$.

an approach can clean up EEG data for accurate pinpointing the center of epilepsy as proven by Thatcher et al. [17]. This cleaned up version will be useful for a home-based patient monitoring system for epileptic seizures and other cognitive measures.

In this paper, we have jointly addressed the possibility of real-time in situ EEG measurement using CS firmware technology intended for out-of-clinic applications. However, to complete the design of video EEG, additional challenges remain such as (i) need for fuzzy logic-based strategy for optimal, nonuniform placement of electrodes that generates stronger SNR for natural sparseness, (ii) a lower-power mixed-signal processor at each electrode (denoise, $A / D$, etc.), and (iii) new advanced system design using an 
Original plot, $0.019531 \mathrm{~s}$

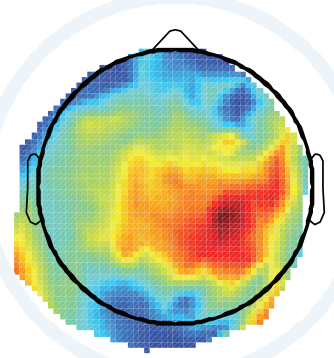

Original plot, $0.027344 \mathrm{~s}$

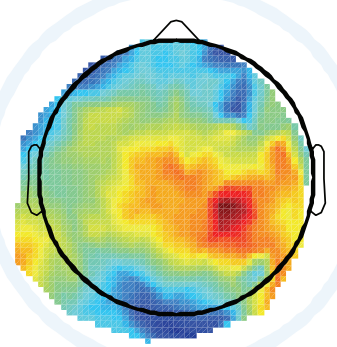

Original plot, $0.066406 \mathrm{~s}$

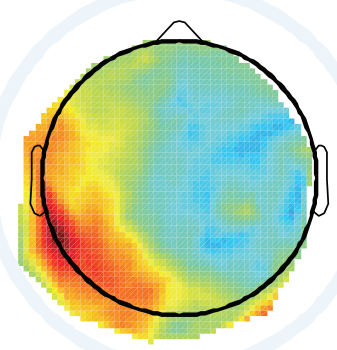

Original plot, $0.089844 \mathrm{~s}$

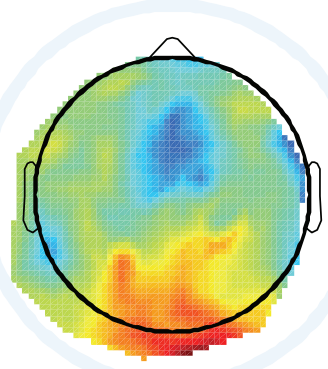

CS plot, $0.019531 \mathrm{~s}$

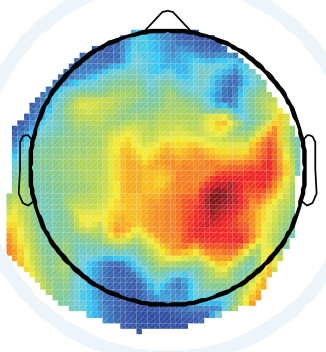

CS plot, $0.027344 \mathrm{~s}$

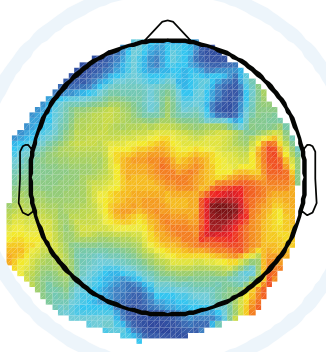

CS plot, $0.066406 \mathrm{~s}$

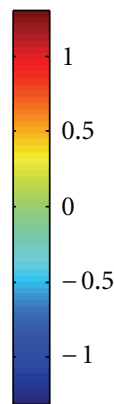

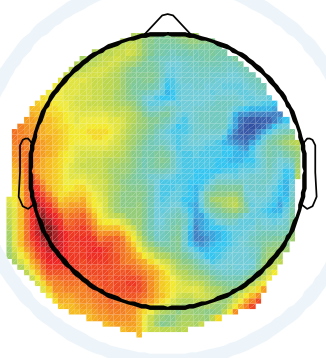

CS plot, $0.089844 \mathrm{~s}$
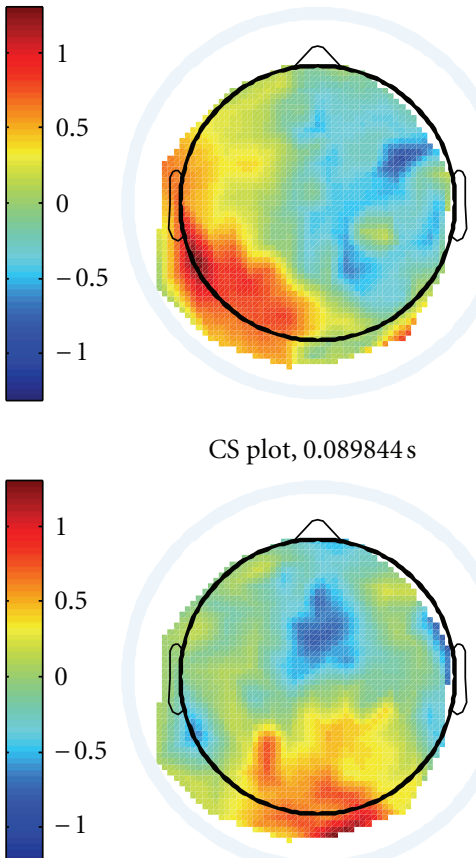

Difference b/w original and CS, $0.019531 \mathrm{~s}$
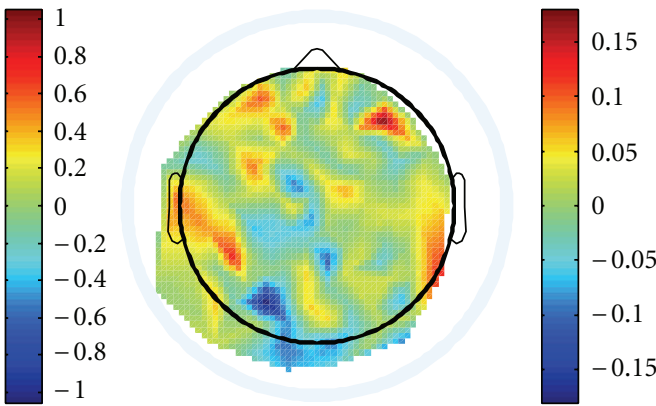

Difference b/w original and CS, $0.027344 \mathrm{~s}$
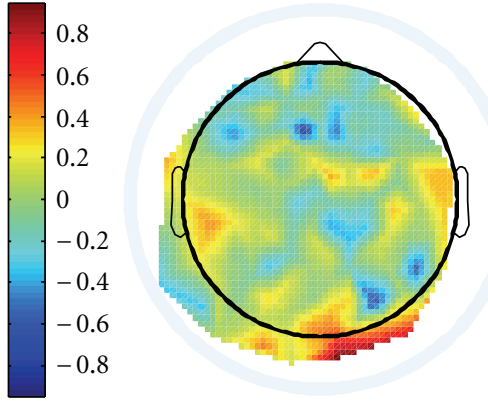

0.2

0.15

0.1

0.05

0

$-0.05$

$-0.1$

$-0.15$

$-0.2$
Difference b/w original and CS, $0.066406 \mathrm{~s}$
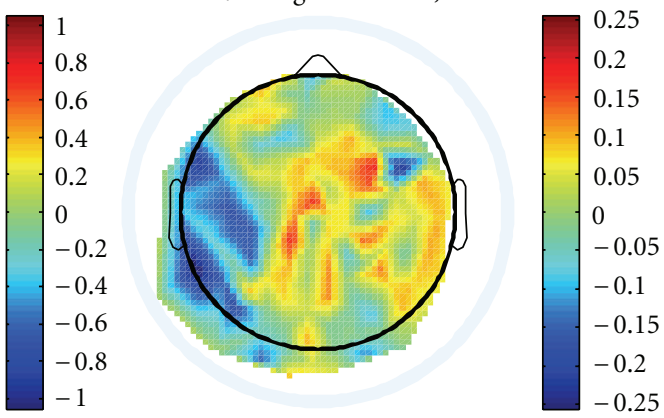

Difference b/w original and CS, $0.089844 \mathrm{~s}$

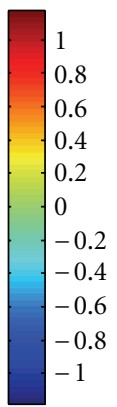

0.2

0.15

0.1

0.05

0

$-0.05$

$-0.1$

$-0.15$

FIGURE 8: Comparison of clinic-based EEG data versus CS algorithm. Number of frames: $256(1 \mathrm{sec}$ ) $, M=32, N=128$, compression ratio $=$ $4: 1$.

ultra-high-density distribution of 500 electrodes adaptively on-off switch of 128 locally. Additional technological challenges, include electromagnetic interference affecting wireless transmission, spatiotemporal delays, and computational speed required by real-time in situ processing.

\section{Acknowledgments}

Original data was taken by a traditional tethered EEG head mount, sponsored by ONR and provided by Dr. Yijun Wang of Swartz Center of UCSD. T. Yamakawa wishes to acknowledge a partial support (Project no. 20001008) of 
de-EMG and de-EMI EEG data called ECoG data for pinpointing the epileptic center entitled "Identification of Epileptogenic Focus by Employing Softcomputing and Establishment of Minimally Invasive and Definitive Surgery" from June 2008 to March 2012 [17].

\section{References}

[1] Y. Wang and T. P. Jung, "A collaborative brain-computer interface for improving human performance," PLOS ONE, vol. 6, no. 5, article e20422, 2011.

[2] Y. T. Wang, Y. Wang, and T. P. Jung, "A cell-phone-based braincomputer interface for communication in daily life," Journal of Neural Engineering, vol. 8, no. 2, Article ID 025018, 2011.

[3] E. J. Candès, J. Romberg, and T. Tao, "Robust uncertainty principles: exact signal reconstruction from highly incomplete frequency information," IEEE Transactions on Information Theory, vol. 52, no. 2, pp. 489-509, 2006.

[4] E. J. Candes and T. Tao, "Near-optimal signal recovery from random projections: universal encoding strategies?" IEEE Transactions on Information Theory, vol. 52, no. 12, pp. 5406-5425, 2006.

[5] D. L. Donoho, "Compressed sensing," IEEE Transactions on Information Theory, vol. 52, no. 4, pp. 1289-1306, 2006.

[6] H. Szu, C. Hsu, J. Jenkins, J. Willey, and J. Landa, "Capturing significant events with neural networks," Neural Networks Journal, vol. 29-30, pp. 1-7, 2012.

[7] F. Pauri, F. Pierelli, G. E. Chatrian, and W. W. Erdly, "Longterm EEG-video-audio monitoring: computer detection of focal EEG seizure patterns," Electroencephalography and Clinical Neurophysiology, vol. 82, no. 1, pp. 1-9, 1992.

[8] A. J. Gabor and M. Seyal, "Automated interictal EEG spike detection using artificial neural networks," Electroencephalography and Clinical Neurophysiology, vol. 83, no. 5, pp. 271-280, 1992.

[9] H. Qu and J. Gotman, "A seizure warning system for long-term epilepsy monitoring," Neurology, vol. 45, no. 12, pp. 2250-2254, 1995.

[10] G. Bauer, F. Aichner, and U. Mayr, "Nonconvulsive status epilepticus followed generalized tonic-clonic seizures," European Neurology, vol. 21, no. 6, pp. 411-419, 1982.

[11] H. Doose, "Nonconvulsive status epilepticus in childhood: clinical aspects and classfication," Advances in Neurology, vol. 34, pp. 115-125, 1984.

[12] C. F. Reynolds, D. J. Kupfer, P. R. Houck et al., "Reliable discrimination of elderly depressed and demented patients by electroencephalographic sleep data," Archives of General Psychiatry, vol. 45, no. 3, pp. 258-264, 1988.

[13] G. C. Blackhart, J. A. Minnix, and J. P. Kline, "Can EEG asymmetry patterns predict future development of anxiety and depression? A preliminary study," Biological Psychology, vol. 72, no. 1, pp. 46-50, 2006.

[14] G. Morabito, A. Bramanti, D. Labate, F. La Foresta, and F. C. Morabito, "Early detection of Alzheimer's onset with Permutation Entropy analysis of EEG," NATO, vol. 1, pp. 30-32, 2011.

[15] C. Bandt and B. Pompe, "Permutation entropy: a natural complexity measure for time series," Physical Review Letters, vol. 88, no. 17, Article ID 174102, 2002.

[16] M. T. Tebano, M. Cameroni, G. Gallozzi et al., "EEG spectral analysis after minor head injury in man," Electroencephalography and Clinical Neurophysiology, vol. 70, no. 2, pp. 185-189, 1988.
[17] R. W. . Thatcher, R. A. Walker, I. Gerson, and F. H. Geilser, "EEG discriminant analysis of mild head trauma," Electroencephalography and Clinical Neurophysiology, vol. 73, pp. 94-106, 1989.

[18] R. W. Thatcher, D. S. Cantor, R. McAlaster, F. Geisler, and P. Krause, "Comprehensive predictions of outcome in closed head-injured patients. The development of prognostic equations," Annals of the New York Academy of Sciences, vol. 620, pp. 82-101, 1991. 

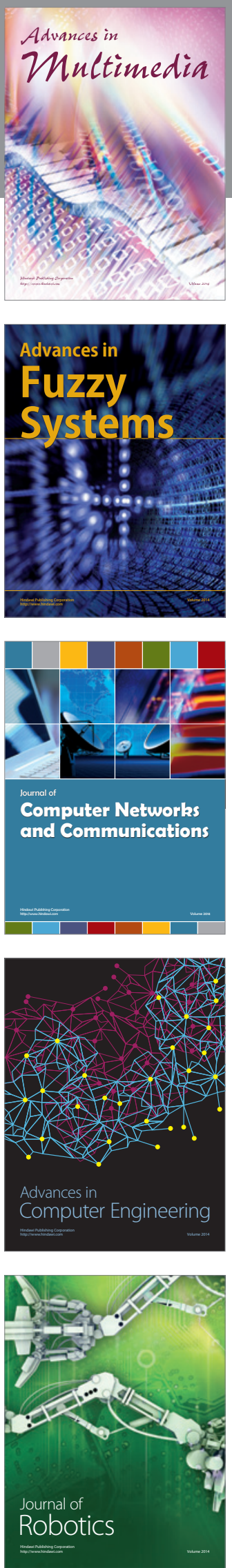

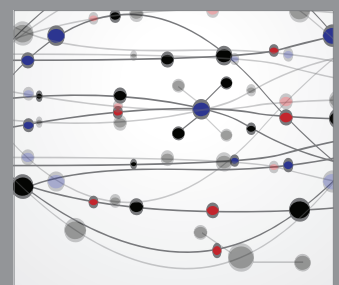

The Scientific World Journal
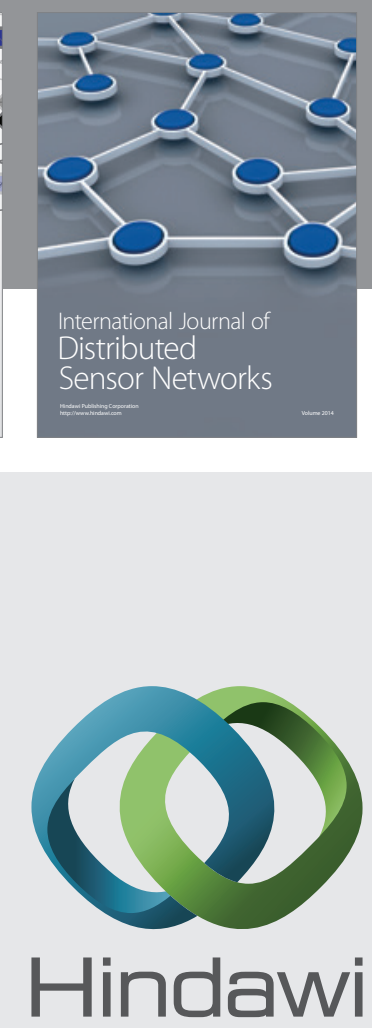

Submit your manuscripts at

http://www.hindawi.com
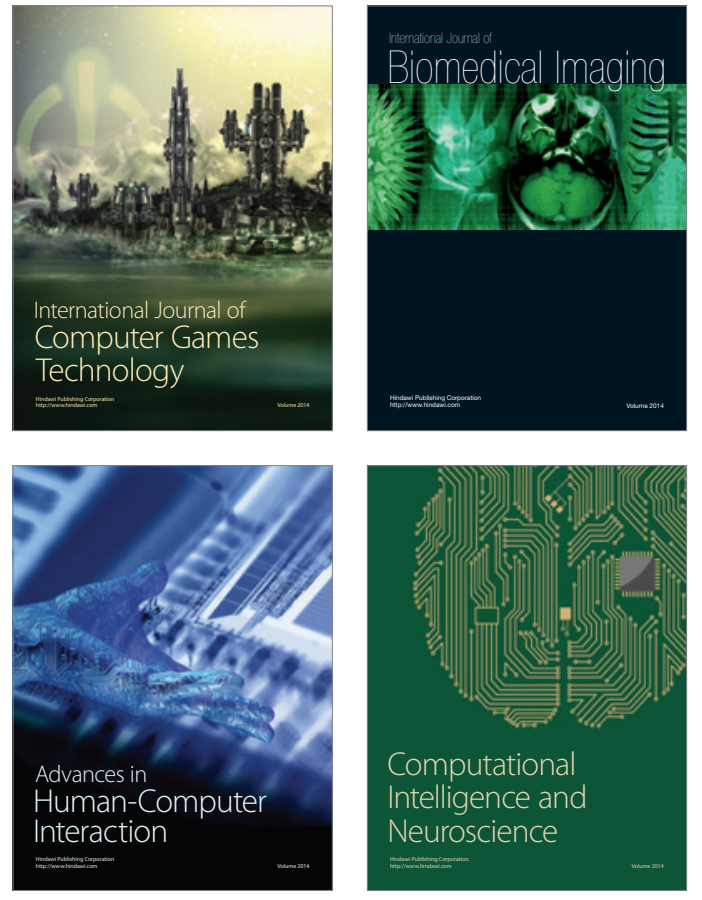
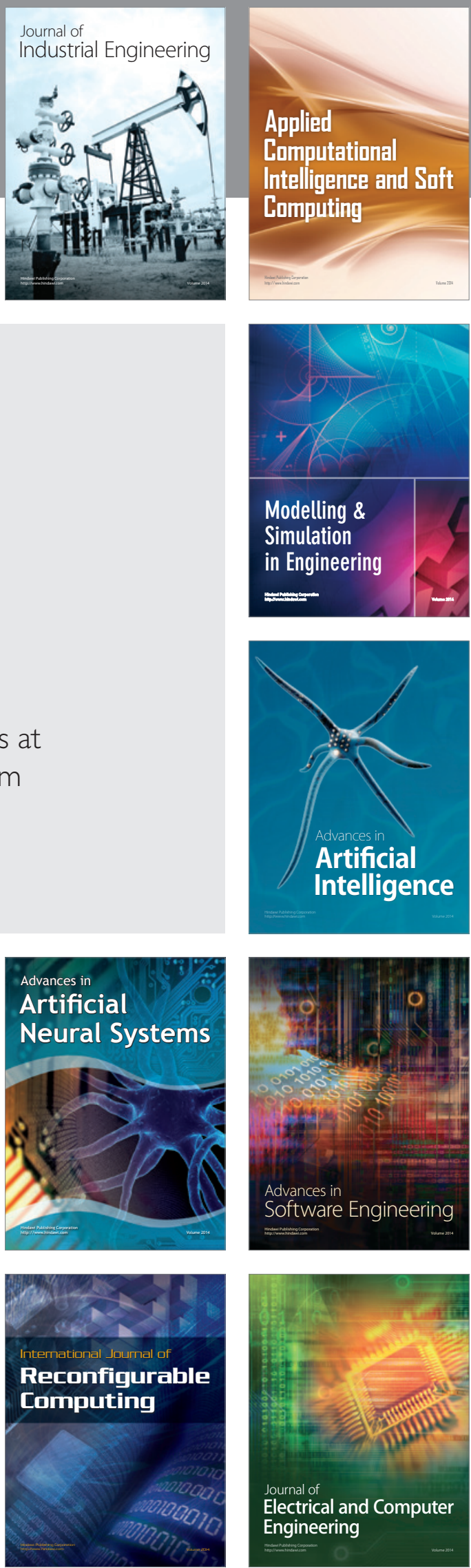